\title{
RKKY interactions and anomalous Hall effect in metallic rare-earth pyrochlores
}

\author{
SungBin Lee, ${ }^{1}$ Arun Paramekanti, ${ }^{1,2}$ and Yong Baek Kim ${ }^{1,2,3}$ \\ ${ }^{1}$ Department of Physics, University of Toronto, Toronto, Ontario M5S 1A7, Canada \\ ${ }^{2}$ Canadian Institute for Advanced Research, Toronto, Ontario, M5G 1Z8, Canada \\ ${ }^{3}$ School of Physics, Korea Institute for Advanced Study, Seoul 130-722, Korea
}

(Dated: June 12, 2021)

\begin{abstract}
Motivated by experiments on $\operatorname{Pr}_{2} \mathrm{Ir}_{2} \mathrm{O}_{7}$, we consider metallic pyrochlore systems $\mathrm{A}_{2} \mathrm{~B}_{2} \mathrm{O}_{7}$, where the A-sites are occupied by rare-earth local moments and the B-sites host $5 d$ transition metal ions with itinerant strongly spin-orbit coupled electrons. Assuming non-Kramers doublets on the Asite, we derive the RKKY interaction between them mediated by the B-site itinerant electrons and find extended non-Heisenberg interactions. Analyzing a simplified model of the RKKY interaction, we uncover a local moment phase with coexisting spiral Ising-like magnetic dipolar and XY-like quadrupolar ordering. This state breaks time-reversal and lattice symmetries, and reconstructs the B-site electronic band structure, producing a Weyl Metallic phase with an intrinsic anomalous Hall effect and an undetectably small magnetization. We discuss implications of our results for $\operatorname{Pr}_{2} \operatorname{Ir}_{2} \mathrm{O}_{7}$.
\end{abstract}

The metallic pyrochlores, $\mathrm{A}_{2} \mathrm{~B}_{2} \mathrm{O}_{7}$, with a rare-earth A-site ion and a $5 d$ transition metal B-site ion, lie at the intersection of exciting recent developments in condensed matter physics. The rare-earth moments on the A-site pyrochlore sublattice could lead to quantum spin ice physics [1, 2] from Ising anisotropy and geometric frustration, a feature they share with the well-studied insulating pyrochlore oxides. The strongly spin-orbit coupled $5 d$ conduction electrons, on the other hand, contain the seeds of topological phases like Weyl semimetals or topological insulators 3 . 8 . The interplay of these two effects could pave the way for new emergent phenomena. The pyrochlore iridate $\mathrm{Pr}_{2} \mathrm{Ir}_{2} \mathrm{O}_{7}$ provides an example of such a metallic frustrated system, with a significant CurieWeiss temperature $\theta_{C W} \approx-20 \mathrm{~K}$, much larger than in the insulating compounds $\mathrm{Pr}_{2} \mathrm{Sn}_{2} \mathrm{O}_{7}$ or $\mathrm{Pr}_{2} \mathrm{Ti}_{2} \mathrm{O}_{7}$ [9]12. For $T \lesssim 1.7 \mathrm{~K}$, even when an applied magnetic field along the $\langle 111\rangle$ direction is switched off, it exhibits a significant anomalous Hall effect (AHE) which grows upon cooling [13 15, although, in contrast to $\mathrm{Nd}_{2} \mathrm{Mo}_{2} \mathrm{O}_{7}$, the uniform magnetization is undetectably small over a range of temperatures. [16] This raises the key issue of mechanisms underlying the AHE [17] in materials with geometric frustration and strong spin-orbit coupling.

For metallic pyrochlores, the RKKY interaction between the local $f$-moments induced by the conduction electrons is expected to be important. When the $f$ moment is a non-Kramers doublet, such as in $\operatorname{Pr}^{3+}$, the pseudospin component $\tau^{z}$ along the local $\langle 111\rangle$ direction carries a magnetic dipole moment and couples to the conduction electron spin density, while the $\tau^{x, y}$ components carry a quadrupole moment and couple to the electronic charge density. We show that the resulting RKKY coupling has a highly non-Heisenberg form and extends beyond the nearest neighbor term. We propose and study a simplified such extended XXZ model for non-Kramers doublets using a variational analysis, finding a rich phase diagram, shown in Fig. 1. which includes incommensurate spiral 'coexistence' states $\left(\mathrm{CE}_{1}, \mathrm{CE}_{2}\right)$, with wavevec-

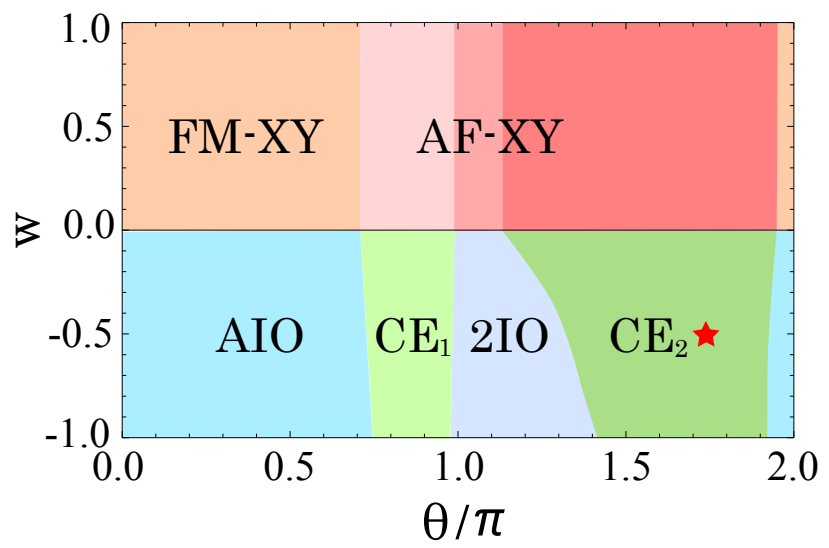

FIG. 1: Variational phase diagram of the pseudospin Hamiltonian in Eq. 22 relevant to RKKY coupled non-Kramers doublets as a function of anisotropy $w$ and $\theta=\tan ^{-1}\left(J_{2} / J_{1}\right)$. $\underline{w}>0$ : We find ferro-XY (FM-XY), antiferro-XY (AF-XY), where spins order in the plane transverse to the local $\langle 111\rangle$ axes. These are time-reversal invariant states with modulated quadrupolar order. $w<0$ : We find Ising all-in/out (AIO), Ising two-in/out (2IO) and states with coexisting modulated Ising-XY orders $\left(\mathrm{CE}_{1}, \mathrm{CE}_{2}\right)$. The $\mathrm{CE}_{2}$ state breaks timereversal and lattice symmetries, producing an anomalous Hall effect for the spin-orbit coupled conduction electrons.

tors $(0, q, \pi)$ or $(0,0, q)$, having spatially modulated, magnetic (dipolar) and quadrupolar order, i.e., 'magnetoquadrupolar supersolids'. We confirm that such a $\mathrm{CE}_{2}$ state also exists within a classical Monte Carlo simulation. Although the $\mathrm{CE}_{2}$ state has no net local moment magnetization, it reconstructs the band structure of the spin-orbit coupled $5 d$-electrons, producing Weyl points as well as small Fermi pockets, leading to a measurable AHE and an extremely small conduction electron magnetization [29]. We discuss possible implications and predictions for $\operatorname{Pr}_{2} \mathrm{Ir}_{2} \mathrm{O}_{7}$, for which our proposal appears to be distinct from the more widely discussed spin chirality scenario [15, 18, 20]. 
Basic microscopics and RKKY coupling.- Since we are motivated by experiments on $\operatorname{Pr}_{2} \operatorname{Ir}_{2} \mathrm{O}_{7}$, we briefly review the relevant microscopics of the local moments and conduction electrons in this material. The $\mathrm{Pr}^{3+}$ ion is in a $4 f^{2}$ configuration, with electron-electron interactions and strong spin-orbit coupling favoring a total angular momentum state with $J=4$. This ninefold degenerate manifold is split by crystal fields with $D_{3 d}$ point group symmetry around the $\mathrm{Pr}^{3+}$ ion, arising from a cage of eight $\mathrm{O}^{2-}$ ions stretched along the local $\langle 111\rangle$ axis. The splitting is captured by an effective time-reversal invariant crystal field Hamiltonian expressed in terms of $J=4$ angular momentum operators, $H_{\text {cef }}=-\alpha J_{z}^{2}+\beta\left[J_{z}\left(J_{+}^{3}+J_{-}^{3}\right)+\right.$ h.c. $]+\gamma\left(J_{+}^{6}+J_{-}^{6}\right)$, with $\alpha>0$ and $\beta, \gamma \ll \alpha$, leading to a ground state non-Kramers doublet with a dominant $\left|J_{z}= \pm 4\right\rangle$ component. 21] Projection to this low energy doublet allows us to define pseudospin-1/2 operators $\tau$ with $\tau^{z} \propto J_{z}$ while $\tau^{ \pm} \propto$ $\left\{J_{ \pm}, J_{z}\right\}$. Under time-reversal, $\tau^{z} \rightarrow-\tau^{z}$, transforming as the magnetic dipole moment, while $\tau^{x, y}$ are left invariant, transforming like a quadupole moment.

For $\mathrm{Ir}^{4+}$ electrons, strong spin-orbit coupling in the $t_{2 g}$ manifold leads to a half-filled effective $j=1 / 2$ band. The metallic character of $\operatorname{Pr}_{2} \mathrm{Ir}_{2} \mathrm{O}_{7}$ suggests that a tight binding model which ignores strong electronic correlations would be an adequate starting point,

$$
H_{\mathrm{tb}}=\sum_{i j} \sum_{\alpha \beta} c_{i \alpha}^{\dagger}\left(t_{i j} \delta_{\alpha \beta}+i \boldsymbol{v}_{i j} \cdot \boldsymbol{\sigma}_{\alpha \beta}\right) c_{j \beta},
$$

where $c_{i \alpha}^{\dagger}\left(c_{j \beta}\right)$ denotes the electron creation (annihilation) operator at site $i$ with the Kramers pseudospin index $\alpha$ corresponding to $j_{z}= \pm 1 / 2$, and $\sigma=\left(\sigma^{x}, \sigma^{y}, \sigma^{z}\right)$ are the Pauli matrices. We assume $t_{i j}=t\left(t^{\prime}\right)$ for nearest (next-nearest) neighbors, and $\boldsymbol{v}_{i j} \neq 0$ only for nearestneighbors and is constrained by lattice symmetries. 22]

How does the A-site non-Kramers doublet couple to the B-site conduction electrons? Unlike the usual Kondo coupling to a magnetic Kramers doublet, time-reversal invariance dictates that the Ising component of the A-site pseudospin $\tau_{j}^{z}$ at site $j$ (which points along the local $\langle 111\rangle$ axis) couples to spin density $j_{i}^{\mu}=c_{i \alpha}^{\dagger} \frac{\sigma_{\alpha \beta}^{\mu}}{2} c_{i \beta}$ of electrons on the six neighboring B-sites, while the planar components of the pseudospin $\tau_{j}^{ \pm}$couple to the charge density $n_{i}=c_{i \alpha}^{\dagger} c_{i \alpha}$ on the neighboring B-sites. Keeping $j_{i}^{\mu} \tau_{j}^{z}$ and $n_{i} \tau_{j}^{ \pm}$terms, we find the symmetry allowed Kondo coupling $H_{A B}$ with three parameters $c_{1}, c_{2}, c_{3}$. (See Supplementary Materials for details.) Integrating out the conduction electrons, we find that the resulting RKKY interaction has two important features which are insensitive to the details of the Ir band structure: (i) it allows for significant couplings beyond the nearest-neighbor interaction, but is negligible beyond the third neighbor; (ii) it is highly anisotropic in spin-space since $\tau^{x, y}$ and $\tau^{z}$ interact very differently with the spin-orbit coupled conduction band. A complete microscopic set of Kondo couplings should include $\tau_{j}^{z} c_{i \alpha}^{\dagger} \frac{\sigma_{\alpha \beta}^{\mu}}{2} c_{\ell \beta}$ or $\tau_{j}^{ \pm} c_{i \alpha}^{\dagger} c_{\ell \alpha}$ with $i \neq \ell$, terms which we have omitted, and the full characterization may require multiple parameters. Rather than dealing with such a complex model, we would like to focus on general and robust features of the RKKY interactions. We therefore turn to a study of a simplified RKKY Hamiltonian which retains the two key features described above.

Local Moment Model and Phase Diagram. - Motivated by our observation that the RKKY interaction between local moments is highly anisotropic in spin space, and has beyond nearest-neighbor terms, we study a simplified model in the basis with a sublattice-dependent quantization axis along the local $\langle 111\rangle$ direction,

$$
H=-\sum_{\boldsymbol{r}, \boldsymbol{r}^{\prime}} J_{\boldsymbol{r}, \boldsymbol{r}^{\prime}}\left[(1-w) \tau_{s}^{z}(\boldsymbol{r}) \tau_{s^{\prime}}^{z}\left(\boldsymbol{r}^{\prime}\right)+\boldsymbol{\tau}_{s}^{\perp}(\boldsymbol{r}) \cdot \boldsymbol{\tau}_{s^{\prime}}^{\perp}\left(\boldsymbol{r}^{\prime}\right)\right](2)
$$

Here $J_{\boldsymbol{r}, \boldsymbol{r}^{\prime}}=J_{1}\left(J_{2}\right)$ for nearest (next-nearest) neighbor sites, $\tau_{s}^{z}(\boldsymbol{r})$ is $z$-component of the pseudospin on sublattice $s$ at site $\boldsymbol{r}, \boldsymbol{\tau}_{s}^{\perp}(\boldsymbol{r})$ denotes the transverse component of the pseudospin which lies in the local XY plane, and $w$ quantifies the exchange anisotropy. We set $J_{1}=J \cos \theta$ and $J_{2}=J \sin \theta$, and explore the phase diagram of this model as a function of $(\theta, w)$.

Treating the spins as classical unit vectors, we minimize the energy using a variational ansatz

$$
\boldsymbol{\tau}_{s}(\boldsymbol{r})=d_{s} \hat{\boldsymbol{e}}_{3}+\sqrt{1-d_{s}^{2}} \mathcal{R} e\left[\left(\hat{\boldsymbol{e}}_{1}+i \hat{\boldsymbol{e}}_{2}\right) e^{i\left(\boldsymbol{Q} \cdot \boldsymbol{r}+\varphi_{s}\right)}\right],
$$

where $\hat{e}_{1,2,3}$ form a triad of orthonormal vectors, so that $\left|\boldsymbol{\tau}_{s}(\boldsymbol{r})\right|=1$. In the local coordinate system, this ansatz allows, (i) for $d_{s}=0$, a coplanar spiral with wavevector $\boldsymbol{Q}$ with spins in the $\left(\hat{e}_{1}, \hat{e}_{2}\right)$ plane, and, (ii) for $d_{s}^{2}=1$, a collinear state with spins along $\pm \hat{e}_{3}$. In the isotropic limit, $w=0$, this ansatz recovers $(0,0, q)$ spirals [23], while in the Ising limit it allows for 2-in 2-out or all-in all-out states. The complete ground state phase diagram from this variational analysis is shown in Fig. 1.

For $w>0$, we find states where the spins lie in the local $\mathrm{XY}$ plane, forming phases like ferromagnetic XY, antiferromagnetic XY, or degenerate XY versions of 2-in 2-out states. These XY ordered states for the non-Kramers ions do not break time-reversal symmetry but correspond to modulated quadrupolar orders, with no net quadrupole moment and hence may be termed 'antiferroquadrupolar' (AFQ) states. With increasing $\theta$, the different colors in the AF-XY region represent states with distinct ordering wavevectors $\boldsymbol{Q}=(0 q \pi),(0,0,0),(00 q)$.

For $w<0$, by contrast, we find in addition to well known states like the ferromagnetic Ising (all-in all-out), and Ising spin ice (2-in 2-out), large parameter regimes which support coexistence phases $\left(\mathrm{CE}_{1}, \mathrm{CE}_{2}\right)$ with coplanar order involving spatially modulated Ising (magnetic dipolar) and XY (quadrupolar) order. The $\mathrm{CE}_{1}$ and $\mathrm{CE}_{2}$ states order at the wavevectors $(0, q, \pi)$ and $(0,0, q)$ respectively (or their symmetry related momenta). Such 
CE states are 'magneto-quadrupolar' supersolids which break time-reversal symmetry (defined by $\tau^{z} \rightarrow-\tau^{z}$ ), and most lattice symmetries. Below, we focus on the remarkable physical properties including the AHE of the $\mathrm{CE}_{2}$ state which is (i) robustly present in an unbiased numerical energy minimization using a simulated annealing approach [30, and (ii) stable to the addition of weak perturbations such as $\left(\tau_{i}^{+} \tau_{j}^{+}+\tau_{i}^{-} \tau_{j}^{-}\right)$to the simple Hamiltonian in Eq. 2. A more complete study including such terms which break the local U(1) symmetry will be discussed elsewhere 24.

$A H E$ and Magnetization in the $C E_{2}$ state. - Once the $\mathrm{CE}_{2}$ state is stabilized on the A-site, it imprints effective spatially varying magnetic fields and chemical potentials on the B-site conduction electrons due to the Kondotype coupling between them. For an ordering wave vector $\boldsymbol{Q}$, this mixes electrons with wave vectors $\boldsymbol{k}$ and $\boldsymbol{k}+\boldsymbol{Q}$, leading to a Hamiltonian of the form

$$
H_{\mathrm{tb}}^{B}(\boldsymbol{k})=\left(\begin{array}{cc}
H_{\mathrm{tb}}(\boldsymbol{k}) & H_{A B}(\boldsymbol{Q}) \\
H_{A B}(-\boldsymbol{Q}) & H_{\mathrm{tb}}(\boldsymbol{k}+\boldsymbol{Q})
\end{array}\right)
$$

For the $\mathrm{CE}_{2}$ state in our anisotropic $J_{1}-J_{2}$ pseudospin model, $\boldsymbol{Q}=(00 q)$, and time-reversal symmetry and all lattice symmetries except $C_{2 z}$ ( $\pi$ rotation along $\hat{z}$ direction) are broken. Once these broken symmetries are inherited by the resulting $5 d$ electron band structure, one can argue for an intrinsic AHE. The $C_{2 z}$ symmetry leads to $\sigma_{y z}=\sigma_{x z}=0$ since the current operator $J_{z}$ is invariant under $C_{2 z}$ rotation $(x, y, z) \rightarrow(-x,-y, z)$ while the currents $J_{y}$ and $J_{x}$ change sign. However, $\sigma_{x y}$ remains unchanged under this rotation and can thus be nonzero.

To explicitly compute the Hall conductivity, we assume the tight-binding parameterization for the Ir $5 d$ electrons in Eq.(1), as relevant to $\operatorname{Pr}_{2} \operatorname{Ir}_{2} \mathrm{O}_{7}$, choosing $t=1$, $t^{\prime}=-0.1$, and $\left|\boldsymbol{v}_{i j}\right|=0.2$. These values are close to those determined from the Slater-Koster parameters. 22, Fig.2 shows an example of the reconstructed B-site band structure based on Eq. (4). (Red line indicates the Fermi level.) We consider the A-site incommensurate $\mathrm{CE}_{2}$ local moment order with wave vector $\boldsymbol{Q} \approx 1.2 \pi(001)$, which is appropriate for $\theta=1.72 \pi, w=-0.5$ in Eq. (2) ( $\star$ marked in Fig 1), and the Kondo coupling constant $c / t=0.1$ where $c_{1}=0, c_{2}=c_{3}=c$ in $H_{A B}(\boldsymbol{Q})$. (See Supplementary material) The reconstructed band structure generates both Weyl points and Fermi pockets near $\Gamma, \Gamma+\boldsymbol{Q}$. There are four pairs of Weyl points in total (two pairs near $\Gamma, \Gamma+\boldsymbol{Q}$ and other two pairs at their $C_{2 z}$ symmetry related points) and the inset of Fig 2 shows one of those Weyl points and Fermi pockets near $\boldsymbol{k} \approx(0,0.12 \pi, 0.02 \pi)$.

Fig 3 shows the explicitly calculated Hall conductivity using the Kubo formula as a function of Kondo coupling constant $c$, based on the reconstructed B-site band structures. The strength of Kondo coupling $c$ determines the magnitude of the AHE response. $\sigma_{x y}$ initially increases with increasing $c$, acquiring contributions from small electron-like and hole-like Fermi pockets as well as

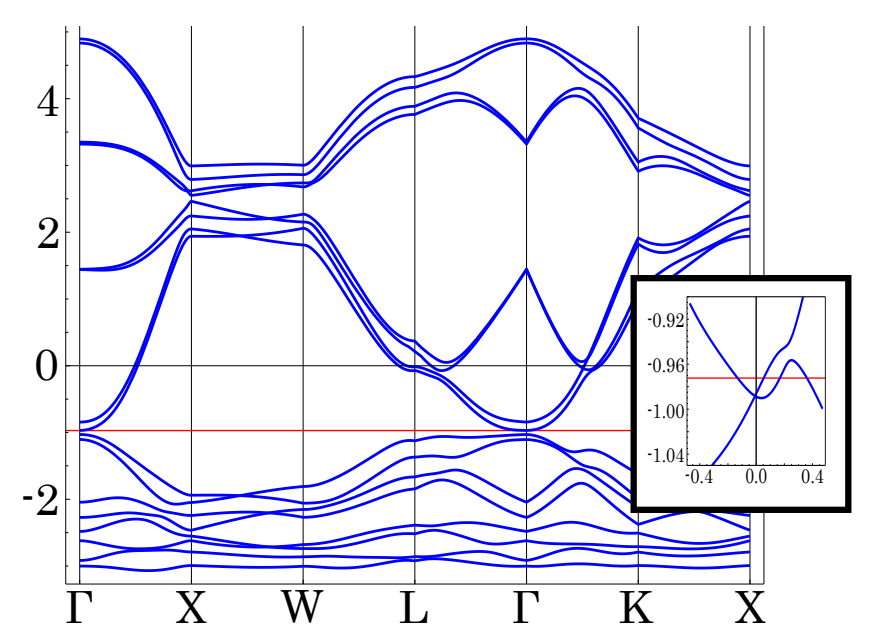

FIG. 2: Reconstructed $5 d$ conduction electron band structure (based on Eq. (4) with the energy units of $t$ ) in the presence of Kondo coupling constant $c / t=0.1$ and the A-site incommensurate $\mathrm{CE}_{2}$ local moment order with wave vector $\boldsymbol{Q} \approx 1.2 \pi(001)$. The inset shows one Weyl point and Fermi pockets near $\boldsymbol{k} \approx(0,0.12 \pi, 0.02 \pi)$ (the horizontal axis represents $k$ along $\boldsymbol{k}=(k, k+0.12 \pi, 0.02 \pi))$. Its pair is located at $\boldsymbol{k} \approx(0.06 \pi, 0.18 \pi, 0)$. Such a pair of Weyl points is near $\Gamma$ point and there is another pair near $\Gamma+\boldsymbol{Q}$. Furthermore, additional two pairs exist at their $C_{2 z}$ symmetry related points. Overall, there are four pairs of Weyl points.

four pairs of Weyl points induced by the $\mathrm{CE}_{2}$ order. For $c / t \gtrsim 0.7$, a band gap opens up and $\sigma_{x y}=0$.

On symmetry grounds, the AHE must be accompanied by a nonzero uniform magnetization, with $M_{x}=M_{y}=0$ but $M_{z} \neq 0$. Remarkably, although the $\mathrm{CE}_{2}$ state has no net magnetization from the local moments, we find that it induces a nonzero magnetization $M_{z}$ for the $5 d$ electrons, where $M_{\mu}=\frac{1}{N}\left\langle\sum_{i} \sum_{\alpha \beta} c_{i \alpha}^{\dagger} \frac{\sigma_{\alpha \beta}^{\mu}}{2} c_{i \beta}\right\rangle$. For small $c$, the net magnetization $M_{z}$ gets larger, proportional to the density of states (DOS) near the Fermi level, with the same sign and a trend qualitatively similar to $\sigma_{x y}$. For large $c / t \gtrsim 0.7$, we find that $M_{z} \neq 0$ although $\sigma_{x y}=0$, signalling a magnetized band insulator.

This dichotomy of a large $\sigma_{x y}$ but a small $M$ can be argued for as follows. On dimensional grounds, the AHE signal $\sigma_{x y} \sim \frac{e^{2}}{h} \Delta k$, where the momentum scale $\Delta k$ must be induced by an effective 'internal magnetic field' $B^{\text {int }}$ due to the spontaneous breaking of time-reversal symmetry 25, 26]. If the $\tau^{x, y}$ order in the $\mathrm{CE}_{2}$ state reconstructs the band structure to produce a small Fermi pocket, with a Fermi wavevector $k_{F}$ and an effective mass $m^{*}$, we expect $\Delta k \sim B^{\text {int }} m^{*} / k_{F}$ resulting in a large $\sigma_{x y}$ due to a small $k_{F}$ Fermi pocket, while the magnetization $M \sim B^{\text {int }} m^{*} k_{F}$ stays small. The Weyl point contribution from splitting a quadratic band touching, with a curvature $m^{* *}$, on the other hand leads to $\Delta k \sim \sqrt{m^{* *} B^{\text {int }}}$ [27], and a magnetization $M \sim B^{\text {int }}$, so that a small $B^{\text {int }}$ again 


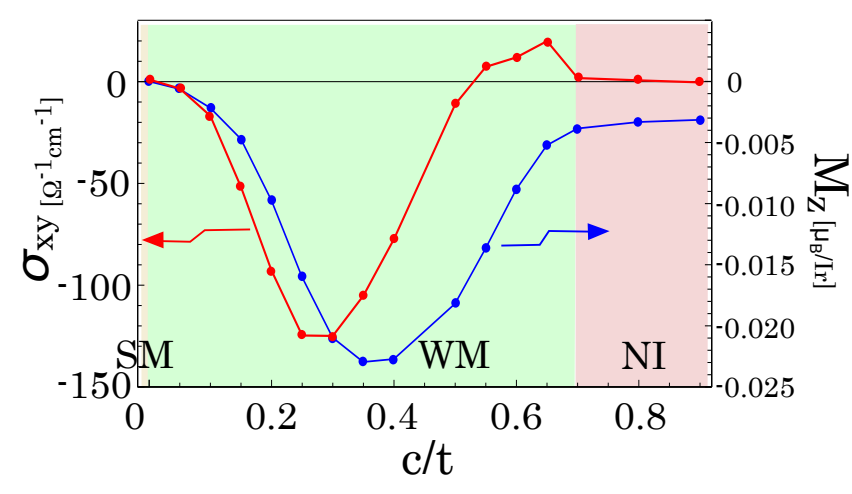

FIG. 3: Hall conductivity $\sigma_{x y}$ and magnetization $M_{z}$ of $5 d$ conduction electrons as a function of Kondo coupling constant $c$ based on the reconstructed band structure. (See Fig 2 for the reconstructed band structure at $c / t=0.1$ ) For $c=0$, the conduction band is in a semi-metal phase (SM) with a quadratic band touching at the $\Gamma$ point. For $0<c / t \lesssim 0.7$, the $\mathrm{CE}_{2}$ state reconstructs the bands generating both Fermi pockets and pairs of Weyl points, resulting in a Weyl-metallic phase (WM), with a significant nonzero $\sigma_{x y}$ which has contributions from Weyl points as well as Fermi surfaces. For $c / t \gtrsim 0.7$, the conduction electrons form a gapped normal insulating phase (NI). The magnetization $M_{z}$ has the same sign as $\sigma_{x y}$ over a wide range of couplings $c$, although it is extremely small in magnitude, and is nonzero even in the NI.

leads to a large $\sigma_{x y}$ and a small $M$. The presence of both contributions would lead to a non-linear $\sigma_{x y}(M)$.

Application to $\mathrm{Pr}_{2} \mathrm{Ir}_{2} \mathrm{O}_{7}$ : The $\mathrm{CE}_{2}$ state in our simple model of RKKY coupled non-Kramers ions and spinorbit coupled conduction electrons captures a key aspect of the experimental data on $\operatorname{Pr}_{2} \mathrm{Ir}_{2} \mathrm{O}_{7}$ : an AHE accompanied by a negligible magnetization. However, since our ordering wavevector is along the $\langle 001\rangle$ direction, the spontaneous AHE is produced in the $x y$ plane, whereas the spontaneous AHE in $\operatorname{Pr}_{2} \mathrm{Ir}_{2} \mathrm{O}_{7}$ is seen for fields along the $\langle 111\rangle$ direction. This discrepancy may be resolved if such coexisting spiral order had a wavevector along the $\langle 111\rangle$ direction, or if our CE order gave way to a multimode spiral formed by superposing $(0,0, q),(0, q, 0),(q, 0,0)$ spirals while preserving $C_{3}$ rotation along $\langle 111\rangle$ direction but breaking all other lattice symmetries. The Hall conductivity in that case will naturally be in the plane perpendicular to $\langle 111\rangle$, in agreement with experiment. We estimate the magnitude of the AHE (at $c / t=0.1$, which is $c \approx 5 \mathrm{meV}$ ) in our $\mathrm{CE}_{2}$ state to be $\sigma_{x y} \approx-15\left(\Omega^{-1} \mathrm{~cm}^{-1}\right)$ and $M_{z} \approx-0.002\left(\mu_{B} / \mathrm{Ir}\right)$. This is consistent with the magnitude of the experimentally measured Hall signal, and the absence of any measurable magnetization over a range of temperatures. At lower temperatures, experiments detect a nonzero remnant magnetization, which may arise in our model from the feedback of the magnetized conduction electrons on the local moments, an effect we have not taken into ac- count.

The Curie-Weiss temperature is sensitive to $J_{2} / J_{1}$, changing sign as a function of $\theta$ even within the $\mathrm{CE}_{2}$ state. For couplings $c / t \sim 0.1$, we can obtain $\theta_{C W}$ to be of the right sign and magnitude, $\theta_{C W} \sim-20 K$ as observed experimentally, for parameter values closer to the all-in all-out phase boundary.

The $\mathrm{CE}_{2}$ state should exhibit two distinct thermal transitions, associated with the onset of Ising and XY orders, with this splitting being smaller for a weaker anisotropy $w$. However, this simple expectation gets confounded by two issues. (i) Terms which we have omitted, such as $\left(\tau_{i}^{+} \tau_{j}^{+}+\tau_{i}^{-} \tau_{j}^{-}\right)$, will break the (staggered) $\mathrm{U}(1)$ invariance of the XY terms, and modify the $3 \mathrm{~d}-\mathrm{XY}$ universality class of the quadrupolar ordering transition. (ii) Oxygen vacancy defects will produce strong electric fields which break the $D_{3 d}$ point group symmetry around the $\mathrm{Pr}^{3+}$ ion, splitting the non-Kramers doublet due to extra random terms $\Delta H_{\text {cef }} \propto\left\{J_{z}, J_{ \pm}\right\} \sim \tau^{ \pm}$in the effective crystal field Hamiltonian. This would lead to a timereversal invariant strong random field on $\tau^{x, y}$. These effects might conspire smear or destroy the XY ordering transition. However, the time-reversal symmetry breaking Ising transition is expected to survive, which would be consistent with the single specific heat "peak" seen at the onset of the spontaneous AHE in $\operatorname{Pr}_{2} \operatorname{Ir}_{2} \mathrm{O}_{7}$. Monte Carlo studies of the thermal properties and disorder effects will be discussed elsewhere 24.

The most direct evidence for the scenario advocated here would be a probe which can detect the modulated Ising order using neutron diffraction. Landau theory arguments predict that entering such a coexistence phase in a clean system would also lead to a weak charge density wave of the Ir electrons at the ordering wavevector of the spiral, from a term $\propto \tau^{x, y}(\boldsymbol{q}) \rho(-\boldsymbol{q})$, and which we find small but finite in our calculations; such charge order may be weak but can be probed, in principle, using $\mathrm{X}$-ray diffraction. In the presence of an induced charge order, Landau theory arguments also predict a nonzero $d$ band magnetization, from a term $\propto M \tau^{z}(\boldsymbol{q}) \rho(-\boldsymbol{q})$, which is indeed present as discussed above. Quantum oscillation measurements to detect the ordering-induced Fermi pockets are desirable, but might be difficult due to the significant AHE and the small size of the pockets.

Conclusion. - In conclusion, we have proposed a mechanism of intrinsic AHE in metallic pyrochlore systems, such as $\operatorname{Pr}_{2} \mathrm{Ir}_{2} \mathrm{O}_{7}$, arising from spiral order of local moments driven by their extended anisotropic RKKY exchange interactions, and the resulting reconstruction of the electronic band structure to form small Fermi pockets and pairs of Weyl points. This ordering could occur proximate to an all-in-all-out state of the local moments, and appears to be distinct from previously proposed spinchirality scenarios for the AHE in $\operatorname{Pr}_{2} \mathrm{Ir}_{2} \mathrm{O}_{7}$.

We are grateful to Subhro Bhattacharjee, Hae-Young Kee, Eric Kin-Ho Lee, Jeffrey Rau for useful discussions. 
This work was supported by NSERC, CIFAR, and Center for Quantum Materials at University of Toronto.

[1] L. Savary and L. Balents, Phys. Rev. Lett 108, 037202 (2012).

[2] S. B. Lee, S. Onoda, and L. Balents, Phys. Rev. B. 86, 104412 (2012).

[3] D. Pesin and L. Balents, Nature Physics 6, 376 (2010).

[4] B.-J. Yang and Y. B. Kim, Phys. Rev. B. 82, 085111 (2010).

[5] X. Wan, A. M. Turner, A. Vishwanath, and S. Y. Savrasov, Phys. Rev. B. 83, 205101 (2011).

[6] W. Witczak-Krempa and Y. B. Kim, Phys. Rev. B. 85, 045124 (2012).

[7] A. Go, W. Witczak-Krempa, G. S. Jeon, K. Park, and Y. B. Kim, Phys. Rev. Lett. 109, 066401 (2012).

[8] X. Wan, A. Vishwanath, and S. Y. Savrasov, Phys. Rev. Lett 108, 146601 (2012).

[9] D. Yanagishima and Y. Maeno, J. Phys. Soc. Jpn 70, 2880 (2001).

[10] K. Matsuhira et al., J. Phys. Soc. Jpn 76, 3706 (2007).

[11] K. Matsuhira et al., J. Phys. Soc. Jpn 71, 1576 (2002).

[12] S. Bramwell, M. Field, M. Harris, and I. Parkin, J. Phys.: Cond. Matt 12, 483 (2000).

[13] S. Nakatsuji et al., Phys. Rev. Lett 96, 087204 (2006).

[14] Y. Machida et al., Phys. Rev. Lett 98, 057203 (2007).

[15] Y. Machida et al., Nature 463, 210 (2009).

[16] Y. Taguchi et al., Science 291, 2573 (2001).

[17] N. Nagaosa et al., Rev. Mod. Phys 82, 1539 (2010).

[18] A. Kalitsov, B. Canals, and C. Lacroix, in J. Phys.: Conf. Ser (IOP Publishing, 2009), vol. 145, p. 012020.

[19] M. Udagawa and R. Moessner, ArXiv e-prints (2012), 1212.0293.

[20] R. Flint and T. Senthil, arXiv preprint arXiv:1301.0815 (2013).

[21] S. Onoda and Y. Tanaka, Phys. Rev. Lett 105, 047201 (2010).

[22] E. K.-H. Lee, S. Bhattacharjee, and Y. B. Kim, arXiv preprint arXiv:1210.5242 (2012).

[23] M. F. Lapa and C. L. Henley, arXiv preprint arXiv:1210.6810 (2012).

[24] S. B. Lee et al., in preparation.

[25] A. Burkov and L. Balents, Phys. Rev. Lett 107, 127205 (2011).

[26] K.-Y. Yang, Y.-M. Lu, and Y. Ran, Phys. Rev. B 84, 075129 (2011).

[27] E.-G. Moon, C. Xu, Y. B. Kim, and L. Balents, arXiv preprint arXiv:1212.1168 (2012).

[28] G. Chen and M. Hermele, Phys. Rev. B. 86, 235129 (2012).

[29] The presence of Fermi pockets does not allow us to seperate the band and Weyl point contributions to $\sigma_{x y}$

[30] Although we have not mapped out the entire parameter space, we find that the variational ansatz captures most of the phases found in the simulated annealing approach. Our ansatz does not capture regimes where multiple- $\boldsymbol{Q}$ orders are favored - such states are well known to occur in the isotropic case $w=0$ in the regime where we find $\mathrm{CE}_{1}$ order, and will be discussed separately in future work for $w \neq 0$ 


\section{Supplementary Material}

\section{Local coordinates and primitive vectors in $\mathbf{A}_{2} \mathbf{B}_{2} \mathbf{O}_{7}$}

First, we define the local coordinates for every A-site pyrochlore lattice. The local spin quantization axis $(\hat{z})$ is always pointing towards the center of each tetrahedron and $\hat{x}, \hat{y}$ axes are defined on its basal plane. Table[ $[$ shows their local coordinates frames depending on the four sublattices of the pyrochlore lattice. The A-site pseudospin in global coordinates, say $\tilde{\boldsymbol{\tau}}_{s}(\boldsymbol{r})$, can be represented as

$$
\tilde{\boldsymbol{\tau}}_{s}(\boldsymbol{r})=\tau_{s}^{x}(\boldsymbol{r}) \hat{x}_{s}+\tau_{s}^{y}(\boldsymbol{r}) \hat{y}_{s}+\tau_{s}^{z}(\boldsymbol{r}) \hat{z}_{s}
$$

where $\boldsymbol{\tau}_{s}(\boldsymbol{r})=\left(\tau_{s}^{x}(\boldsymbol{r}), \tau_{s}^{y}(\boldsymbol{r}), \tau_{s}^{z}(\boldsymbol{r})\right)$ is the pseudospin at site $\boldsymbol{r}$ and sublattice $s$ defined in the local coordinates. Both $\mathrm{A}$ and B-sites form pyrochlore lattice and each can be viewed as FCC lattice with a four-site basis. We define the primitive vectors $\left(b_{i}\right)$ and the position of four sublattices $\left(A_{i}, B_{i}\right)$ for $\mathrm{A}$ and B-sites in Table II

\begin{tabular}{|c|c|c|c|c|}
\hline$i$ & 0 & 1 & 2 & 3 \\
\hline \hline$\hat{x}_{i}$ & $\frac{1}{\sqrt{2}}(01 \overline{1})$ & $\frac{1}{\sqrt{2}}(0 \overline{1} 1)$ & $\frac{1}{\sqrt{2}}(011)$ & $\frac{1}{\sqrt{2}}(0 \overline{1} \overline{1})$ \\
$\hat{y}_{i}$ & $\frac{1}{\sqrt{6}}(\overline{2} 11)$ & $\frac{1}{\sqrt{6}}(\overline{2} \overline{1} \overline{1})$ & $\frac{1}{\sqrt{6}}(21 \overline{1})$ & $\frac{1}{\sqrt{6}}(2 \overline{1} 1)$ \\
$\hat{z}_{i}$ & $\frac{1}{\sqrt{3}}(111)$ & $\frac{1}{\sqrt{3}}(1 \overline{1} \overline{1})$ & $\frac{1}{\sqrt{3}}(\overline{1} 1 \overline{1})$ & $\frac{1}{\sqrt{3}}(\overline{1} \overline{1} 1)$ \\
\hline
\end{tabular}

TABLE I: Local coordinate frames for the four sublattices on the pyrochlore lattice.

\begin{tabular}{|c|c|c|c|c|}
\hline$i$ & 0 & 1 & 2 & 3 \\
\hline \hline$A_{i}$ & $(0,1,0)$ & $\left(0, \frac{3}{2}, \frac{1}{2}\right)$ & $\left(\frac{1}{2}, 1, \frac{1}{2}\right)$ & $\left(\frac{1}{2}, \frac{3}{2}, 0\right)$ \\
$B_{i}$ & $(0,0,0)$ & $\left(0, \frac{1}{2}, \frac{1}{2}\right)$ & $\left(\frac{1}{2}, 0, \frac{1}{2}\right)$ & $\left(\frac{1}{2}, \frac{1}{2}, 0\right)$ \\
$b_{i}$ & & $(0,1,1)$ & $(1,0,1)$ & $(1,1,0)$ \\
\hline
\end{tabular}

TABLE II: Primitive vectors $\left(b_{i}\right)$ and four basis vectors on each A-site $\left(A_{i}\right)$ and B-site $\left(B_{i}\right)$.

\section{Kondo-like coupling between $A$ and B-sites}

In this section, we derive the explicit form of the Kondo-like coupling between the A-site non-Kramers doublet and the B-site Kramers doublet. As we argued in the main text, the Ising component of the A-site pseudospin $\tau_{j}^{z}$ couples to their six neighboring B-site magnetic moments $j_{i}^{\mu}=c_{i \alpha}^{\dagger} \frac{\sigma_{\alpha \beta}^{\mu}}{2} c_{i \beta}$, whereas, the A-site planar components $\tau_{j}^{ \pm}$couple to the B-site electron density $n_{i}=c_{i \alpha}^{\dagger} c_{i \alpha}$. Hence, we separate the former and the latter cases and derive the Kondo-like coupling term allowed by lattice symmetry. First of all, let's consider the former case where $\tau_{j}^{z}$ couples to $j_{i}^{\mu}$. We note that this magnetic coupling term is already derived in Ref.28.

$$
\begin{aligned}
H_{A B}^{z z}=\sum_{r} & c_{1}\left[\left\{j_{1}^{x}(r)+j_{1}^{x}\left(r-b_{2}+b_{3}\right)+j_{2}^{y}\left(r+b_{1}-b_{2}\right)+j_{2}^{y}\left(r-b_{2}+b_{3}\right)+j_{3}^{z}(r)+j_{3}^{z}\left(r+b_{1}-b_{2}\right)\right\} \tau_{0}^{z}(r)\right. \\
& +\left\{j_{0}^{x}\left(r+b_{1}\right)+j_{0}^{x}\left(r+b_{1}-b_{2}+b_{3}\right)-j_{2}^{z}\left(r+b_{1}-b_{2}\right)-j_{2}^{z}\left(r+b_{1}-b_{2}+b_{3}\right)\right. \\
& \left.-j_{3}^{y}\left(r+b_{1}\right)-j_{3}^{y}\left(r+b_{1}-b_{2}\right)\right\} \tau_{1}^{z}(r)+\left\{j_{0}^{y}\left(r+b_{1}\right)+j_{0}^{y}\left(r+b_{3}\right)\right. \\
& \left.-j_{1}^{z}(r)-j_{1}^{z}\left(r+b_{3}\right)-j_{3}^{x}(r)-j_{3}^{x}\left(r+b_{1}\right)\right\} \tau_{2}^{z}(r)+\left\{j_{0}^{z}\left(r+b_{3}\right)+j_{0}^{z}\left(r+b_{1}-b_{2}+b_{3}\right)\right. \\
& \left.\left.-j_{1}^{y}\left(r+b_{3}\right)-j_{1}^{y}\left(r-b_{2}+b_{3}\right)-j_{2}^{x}\left(r-b_{2}+b_{3}\right)-j_{2}^{x}\left(r+b_{1}-b_{2}+b_{3}\right)\right\} \tau_{3}^{z}(r)\right] \\
& +c_{2}\left[\left\{j_{1}^{y}(r)+j_{1}^{y}\left(r-b_{2}+b_{3}\right)+j_{1}^{z}(r)+j_{1}^{z}\left(r-b_{2}+b_{3}\right)+j_{2}^{x}\left(r+b_{1}-b_{2}\right)+j_{2}^{x}\left(r-b_{2}+b_{3}\right)\right.\right. \\
& \left.+j_{2}^{z}\left(r+b_{1}-b_{2}\right)+j_{2}^{z}\left(r-b_{2}+b_{3}\right)+j_{3}^{x}(r)+j_{3}^{x}\left(r+b_{1}-b_{2}\right)+j_{3}^{y}(r)+j_{3}^{y}\left(r+b_{1}-b_{2}\right)\right\} \tau_{0}^{z}(r) \\
& +\left\{-j_{0}^{y}\left(r+b_{1}\right)-j_{0}^{y}\left(r+b_{1}-b_{2}+b_{3}\right)-j_{0}^{z}\left(r+b_{1}\right)-j_{0}^{z}\left(r+b_{1}-b_{2}+b_{3}\right)\right. \\
& +j_{2}^{x}\left(r+b_{1}-b_{2}\right)+j_{2}^{x}\left(r+b_{1}-b_{2}+b_{3}\right)-j_{2}^{y}\left(r+b_{1}-b_{2}\right)-j_{2}^{y}\left(r+b_{1}-b_{2}+b_{3}\right)+j_{3}^{x}\left(r+b_{1}\right) \\
& \left.+j_{3}^{x}\left(r+b_{1}-b_{2}\right)-j_{3}^{z}\left(r+b_{1}\right)-j_{3}^{z}\left(r+b_{1}-b_{2}\right)\right\} \tau_{1}^{z}(r)+\left\{-j_{0}^{x}\left(r+b_{1}\right)-j_{0}^{x}\left(r+b_{3}\right)\right. \\
& -j_{0}^{z}\left(r+b_{1}\right)-j_{0}^{z}\left(r+b_{3}\right)-j_{1}^{x}(r)-j_{1}^{x}\left(r+b_{3}\right)+j_{1}^{y}(r)+j_{1}^{y}\left(r+b_{3}\right) \\
& \left.+j_{3}^{y}(r)+j_{3}^{y}\left(r+b_{1}\right)-j_{3}^{z}(r)-j_{3}^{z}\left(r+b_{1}\right)\right\} \tau_{2}^{z}(r)+\left\{-j_{0}^{x}\left(r+b_{3}\right)-j_{0}^{x}\left(r+b_{1}-b_{2}+b_{3}\right)\right. \\
& -j_{0}^{y}\left(r+b_{3}\right)-j_{0}^{y}\left(r+b_{1}-b_{2}+b_{3}\right)-j_{1}^{x}\left(r+b_{3}\right)-j_{1}^{x}\left(r-b_{2}+b_{3}\right)+j_{1}^{z}\left(r+b_{3}\right)+j_{1}^{z}\left(r-b_{2}+b_{3}\right) \\
& \left.\left.-j_{2}^{y}\left(r-b_{2}+b_{3}\right)-j_{2}^{y}\left(r+b_{1}-b_{2}+b_{3}\right)+j_{2}^{z}\left(r-b_{2}+b_{3}\right)+j_{2}^{z}\left(r+b_{1}-b_{2}+b_{3}\right)\right\} \tau_{3}^{z}(r)\right]
\end{aligned}
$$

Here, $\tau_{i}^{z}(r)$ is the Ising component of the pseudospin at sublattice $i(=0,1,2,3)$ in unit cell labeled by $r$ which is pointing along local $\hat{z}_{i}$ direction. (See Table $\mathrm{I}$ for their 
local coordinate frames for the four sublattices on A-site pyrochlore lattice) In a similar way, $j_{i}^{\mu}(r)$ is $\mu(=x, y, z)$ component of B-site magnetic moments in global cubic coordinates. Primitive vectors $\left(b_{i}\right)$ and four basis vectors on each A-site $\left(A_{i}\right)$ and B-site $\left(B_{i}\right)$ are shown in Table II. There are two independent parameters, $c_{1}$ and $c_{2}$ which can not be determined by symmetry grounds. However, two independent parameters can be easily understood as follows. For every given site $i$ (on the A-site), there are one local $\hat{z}_{i}$ axis and its basal plane formed by $\hat{x}_{i}$ and

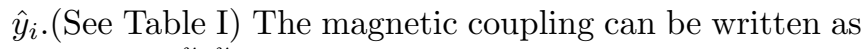
$\tau_{i}^{z}\left(c_{j}^{x} j_{j}^{x}+c_{j}^{y} j_{j}^{y}+c_{j}^{z} j_{j}^{z}\right)$ assuming that $j_{j}^{z}$ is aligned along their local $\hat{z}_{i}$ axis. For its basal plane, we have freedom to choose $\hat{x}_{j}$ and $\hat{y}_{j}$, which leaves $c_{j}^{x}=c_{j}^{y}$. Once the coupling between A-site $i$ and one of their six neighboring B-site $j$ is determined, the coupling with all the other five neighbors are determined by lattice $c_{3}$ rotation and $\sigma_{h}$ mirror symmetries, with two independent parameters $c_{j}^{z}$ and $c_{j}^{x}=c_{j}^{y}$.

On the other hand, the planar components of the Asite pseudospin are time reversal invariant and they do not couple to B-site magnetic moments but couple to the B-site electron density $n_{i}=c_{i \alpha}^{\dagger} c_{i \alpha}$. In this case, there is only one independent parameter and one can write the coupling term as following

$$
\begin{aligned}
H_{A B}^{ \pm}=c_{3} & \sum_{r}\left[\tau _ { 0 } ^ { + } ( r ) \left\{\omega^{2} n_{2}\left(b_{1}-b_{2}+r\right)+\omega^{2} n_{2}\left(-b_{2}+b_{3}+r\right)+\omega n_{3}\left(b_{1}-b_{2}+r\right)+\omega n_{3}(r)\right.\right. \\
& \left.+n_{1}\left(-b_{2}+b_{3}+r\right)+n_{1}(r)\right\}+\tau_{1}^{+}(r)\left\{\omega^{2} n_{3}\left(b_{1}+r\right)+\omega^{2} n_{3}\left(b_{1}-b_{2}+r\right)\right. \\
& \left.+\omega n_{2}\left(b_{1}-b_{2}+r\right)+\omega n_{2}\left(b_{1}-b_{2}+b_{3}+r\right)+n_{0}\left(b_{1}+r\right)+n_{0}\left(b_{1}-b_{2}+b_{3}+r\right)\right\} \\
& +\tau_{2}^{+}(r)\left\{\omega^{2} n_{0}\left(b_{1}+r\right)+\omega^{2} n_{0}\left(b_{3}+r\right)+\omega n_{1}\left(b_{3}+r\right)+n_{3}\left(b_{1}+r\right)+\omega n_{1}(r)+n_{3}(r)\right\} \\
& +\tau_{3}^{+}(r)\left\{\omega^{2} n_{1}\left(b_{3}+r\right)+\omega^{2} n_{1}\left(-b_{2}+b_{3}+r\right)+\omega n_{0}\left(b_{3}+r\right)+\omega n_{0}\left(b_{1}-b_{2}+b_{3}+r\right)\right. \\
& \left.\left.+n_{2}\left(-b_{2}+b_{3}+r\right)+n_{2}\left(b_{1}-b_{2}+b_{3}+r\right)\right\}+h . c\right]
\end{aligned}
$$

Extra phases $1, \omega=e^{i 2 \pi / 3}, \omega^{2}$ are present depending on the different sublattices due to their local coordinates on the A-site pseudospin. Combining $H_{A B}^{z z}$ and $H_{A B}^{ \pm}$, the Kondo-like coupling with three independent coupling parameters $c_{1}, c_{2}, c_{3}$ is written as

$$
H_{A B}\left(c_{1}, c_{2}, c_{3}\right)=H_{A B}^{z z}\left(c_{1}, c_{2}\right)+H_{A B}^{ \pm}\left(c_{3}\right) .
$$

In the presence of magnetic ordering on the A-site, we take $\boldsymbol{\tau}_{s}(r) \rightarrow\left\langle\boldsymbol{\tau}_{s}(r)\right\rangle$ which acts as an effective magnetic field or chemical potential at every B-site itinerant electrons. 